\title{
Field Experiments with the Ames Marsokhod Rover
}

\author{
Daniel Christian ${ }^{1}$, David Wettergreen, Maria Bualat, \\ Kurt Schwehr ${ }^{2}$, Deanne Tucker, Eric Zbinden ${ }^{1}$ \\ Intelligent Mechanisms Group \\ NASA Ames Research Center, MS 269-3 \\ Moffett Field, CA 94035-1000 USA
}

\begin{abstract}
In an ongoing series of field experiments, the Ames Marsokhod rover is deployed to remote locations and operated by scientists in simulated planetary explorations. These experiments provide insight both for scientists preparing for real planetary surface exploration and for robotics researchers. In this paper we will provide an overview of our work with the Marsokhod, describe the various subsystems that have been developed, discuss the latest in a series of field experiments, and discuss the lessons learned about performing remote geology.
\end{abstract}

\section{Introduction}

A terrestrial geologist investigates an area by systematically moving among and inspecting surface features, such as outcrops, boulders, contacts, and faults. A planetary geologist must explore remotely and use a robot to approach and image surface features. Close-up inspection of remote planetary surfaces is a key part of understanding the geological processes at work in our Solar System. Upcoming NASA missions, as well as the recent Mars Pathfinder landing with the Sojourner robot, offer planetary scientists opportunities to use mobile robots to make close observations of surface features, and to help answer long-standing questions regarding planetary formation.

The Intelligent Mechanisms Group (IMG) at NASA Ames Research Center (ARC) has been developing capabilities and control systems in order to research and evaluate the scientific exploration of other planetary surfaces. A series of mission simulations have been performed where the robot is located at a remote field site, but controlled from ARC (or other sites). In each test, the abilities of the robot, the efficiency of remote control, and the accuracy of mission simulation have improved.

Initial tests in Kamchatka, Russia in 1993 tested virtual reality remote control of the robot and imagers. The Amboy crater test in California in 1994 investigated remote science team interaction in the presence of significant time delays. The Kilauea Volcano test in Hawaii in 1995 added a manipulator to the vehicle and more advanced control modes [Hine
1995]. The most recent test in the Painted Desert region of Arizona in 1996 further improved sensors, control modes, onboard autonomy, sample handling, and remote science simulation.

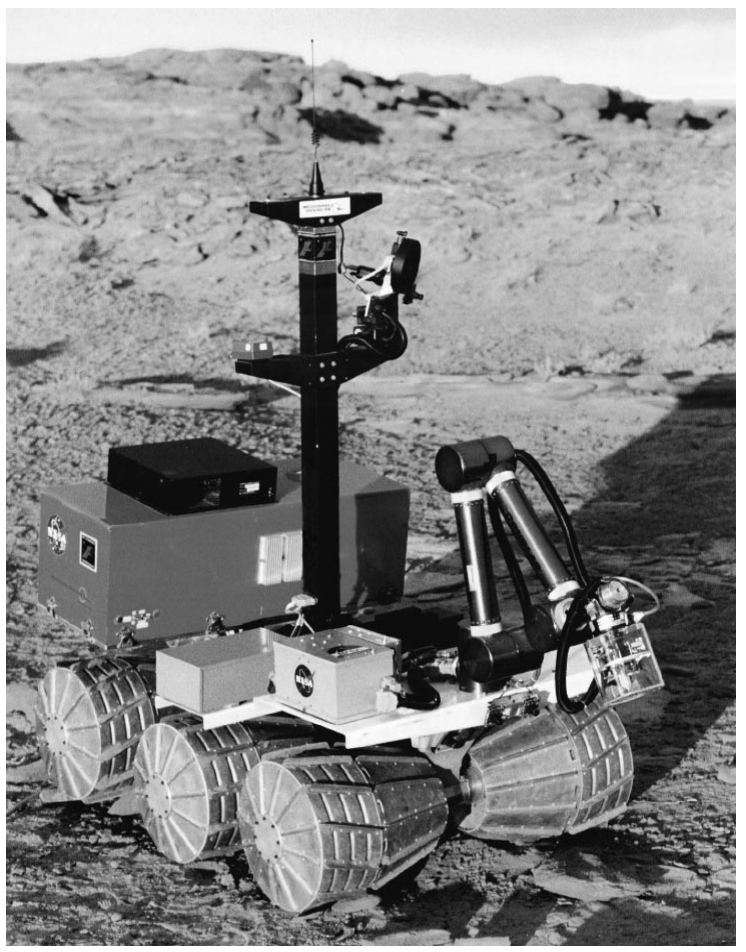

Figure 1: Ames Marsokhod in Arizona

\section{Marsokhod systems}

The research emphasis using Marsokhod (see Figure 1) is on software and sensors for remote exploration. While Marsokhod is larger than the size currently being considered for Mars missions, little or no miniaturization is needed to test many common field instruments as part of the robot. This enables more rapid, inexpensive research into the field effectiveness of different sensor, actuator, and software configurations. Figure 2 shows Marsokhod with a Sojourner model and the Koala micro rover for comparison. 


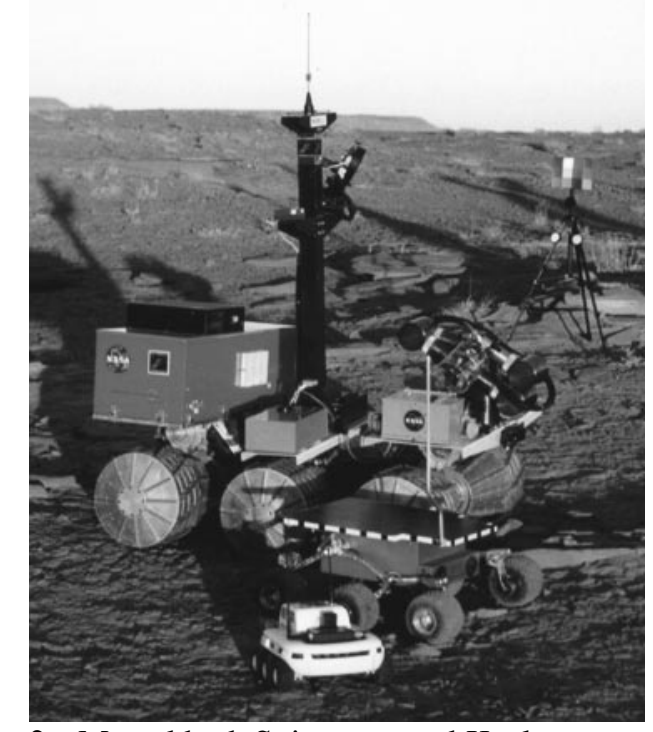

Figure 2: Marsokhod, Sojourner, and Koala

\subsection{Russian built chassis}

The Marsokhod chassis is an all terrain vehicle developed by the Mobile Vehicle Engineering Institute (VNIITransmash) in Russia [Kermurdjian 1992]. The chassis is $100 \mathrm{~cm}$ wide, $150 \mathrm{~cm}$ long, and $35 \mathrm{~kg}$ unloaded mass.

The chassis consists of three pairs of independently driven titanium wheels, joined together by a three degree-of-freedom passively articulated frame. Two degrees-of-freedom allow the frame to twist, while the third allows it to pitch. This design enables the rover to conform passively to very rugged terrain. The shape of the wheels provides low ground pressure and minimizes the risk of high centering the rover by enclosing most of the frame. The amplifiers, motors and batteries are mounted inside the wheels to produce a very low center-of-gravity.

Mounted above each segment is a rigid pallet for mounting additional equipment. The front pallet supports the arm and its electronics. The middle pallet supports electronics for the pan-tilt head and arm force sensor and the mast holding the cameras and antennas. An averaging mechanism keeps the mast at the median angle between the front and rear segments. The rear pallet houses the rest of the electronics and computers. Fully equipped, the robot weighs about $100 \mathrm{~kg}$.

\subsection{Instrument and sampling arm}

An arm with tool carousel mounted on the front pallet allows close up imaging, soil mechanics tests, and sample acquisition. Fixed stereo cameras on the front of the vehicle aid in arm placement.

The arm is a research unit developed by MacDonnell Douglas. It has 5 degrees of freedom (DOF) and has a $1 \mathrm{~m}$ maximum reach.

A rotary carousel has been added which holds three instruments and a sampler inside a 100x100mm clear cylindrical housing. An opening at the bottom of the housing allows access to the target. The carousel is linked to the arm through a 6 axis force sensor enabling the robot to sense contact with the surface or an obstacle (see Figure 3).

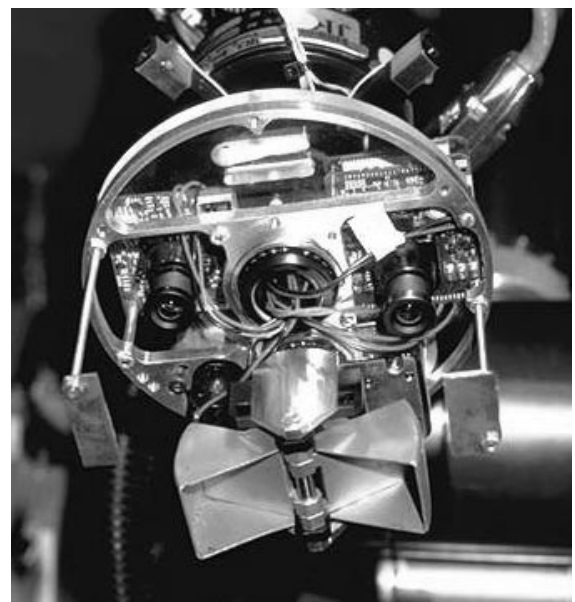

Figure 3: Instrument carousel without housing

Multiple instruments on the carousel can be used without moving the arm. This minimizes disturbance of the site and ensures that the readings from different sensors can be directly correlated. The carousel has instruments in 4 positions: a monochrome camera, a color camera, a clam-shell and an experimental haptic device (not shown). Calibration targets for size and color are in the field of view of each camera.

The resolution of both cameras is $0.08 \mathrm{~mm} / \mathrm{pixel}$ at the housing bottom. The depth of field is approximately $20 \mathrm{~mm}$ and is centered on the housing bottom. The signal from the color camera is converted into separate red, green, and blue signals and digitized independently. Figure 4 shows a monochrome image of the scratch created by the haptic sensor on compacted soil and the $5 \mathrm{~mm}$ calibration stripes.

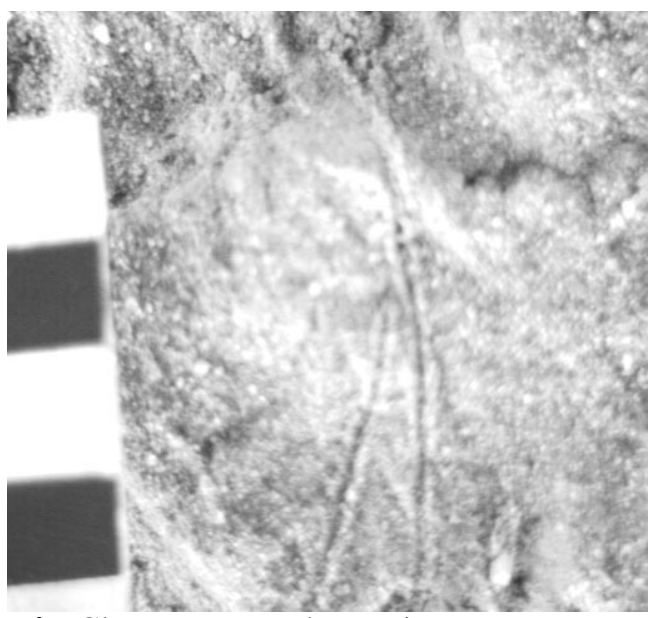

Figure 4: Close-up monochrome image

The clam-shell acquires samples using a single actuator. The clam-shell fits entirely within the housing when the jaws are open and extends outside the housing to acquire soil or pebble samples of up to $12 \mathrm{cc}$. The carousel cannot rotate while the clam-shell is out. The acquired sample can be dropped in a storage container on the front pallet. 


\subsection{Automated arm placement}

It is exceptionally difficult to place the instrument carousel in contact with a surface using cameras alone. The arm force sensor enables contact detection and surface normal estimation for automated carousel placement.

Using the force sensor, the arm can be placed in contact with a surface in as little as two commands. The first moves the arm to a position above the target placement. The second does a guarded move until contact is made. Either move can be a simple joint interpolated move or a cartesian move along a line or about a rotation center.

\subsection{Imaging systems}

The primary imaging hardware consists of a monochrome stereo pair of cameras and a multi-spectral camera mounted about $1.5 \mathrm{~m}$ above the ground. These cameras are mounted on a pan-tilt device with a $318^{\circ}$ pan range and a $111^{\circ}$ tilt range with minimal backlash (see Figure 5).

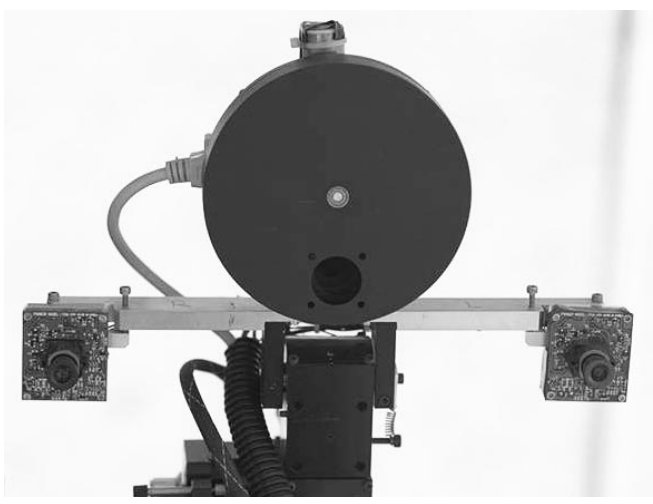

Figure 5: Primary imagers on pan-tilt

All cameras produce monochrome RS-170 format video which is digitized at 512 by 480 resolution by an 8 bit frame grabber. Each camera has independent automatic gain control (AGC) and internal sync generation.

The stereo cameras are inexpensive PC board units with $12 \mathrm{~mm}$ lenses that result in $1 \mathrm{mrad} / \mathrm{pixel}$ resolution. The stereo cameras can be precisely adjusted in roll, pitch, and vergence. The cameras are verged at infinity with zero relative roll and pitch.

The color wheel camera is configured as a low cost simulation of the Imager for Mars Pathfinder (IMP). A monochrome camera with a $12 \mathrm{~mm}$ lens yields $0.7 \mathrm{mrad} / \mathrm{pixel}$ resolution. Figure 6 compares the commercial filters used against the ones on the IMP. Because the camera uses onboard AGC, gray reference targets must be placed in the image to be able to correct the color balance.

A series of tests of the filter wheel were conducted prior to the field test by several geologists familiar with remote sensing. A suite of test rocks were imaged with all filters with direct sun illumination. Although there were color calibration targets in the scene, image saturation made precise color correction impossible. The geologists concluded that the system was useful only for the identification of iron oxide state. The scientists were able to determine if the rock was weathered (oxidized) or had a fresh surface. From these tests it

\begin{tabular}{|r|r|r|r|r|}
\hline Filter & \multicolumn{1}{c|}{$\begin{array}{c}\text { Center } \\
\text { nm }\end{array}$} & $\begin{array}{c}\text { Width } \\
\text { nm }\end{array}$ & $\begin{array}{c}\text { IMP } \\
\text { Center }\end{array}$ & $\begin{array}{c}\text { IMP } \\
\text { Width }\end{array}$ \\
\hline 1 & All pass & & & \\
\hline 2 & 440 & 31 & 440 & 35 \\
\hline 3 & 480 & 30 & 480 & 30 \\
\hline 4 & 530 & 25 & 530 & 30 \\
\hline 5 & 600 & 25 & 600 & 20 \\
\hline 6 & 670 & 20 & 670 & 20 \\
\hline 7 & 750 & 25 & 750 & 20 \\
\hline 8 & 800 & 20 & 800 & 20 \\
\hline
\end{tabular}

Figure 6: Multi-spectral imager vs. IMP imager

became clear that this system of filters is not suited to rock and mineral identification.

The multi-spectral imager is most often used in the $440 \mathrm{~nm}$ (blue), 530nm (green), and 670nm (red) bands to assemble color images or panoramas. Also, the narrow band images provide higher resolution and contrast than the stereo cameras (see Figure 7).

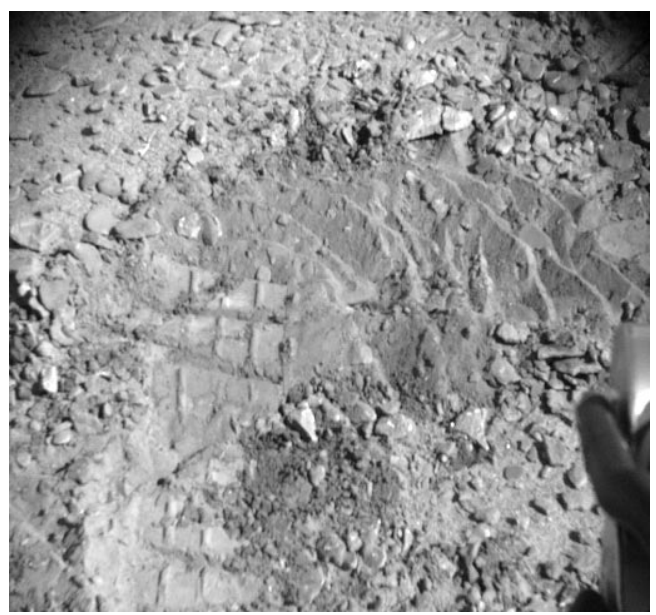

Figure 7: 440nm band image of vehicle tracks

Four types of images are produced: mono images, stereo pairs, mono panoramas, and stereo panoramas. Mono images

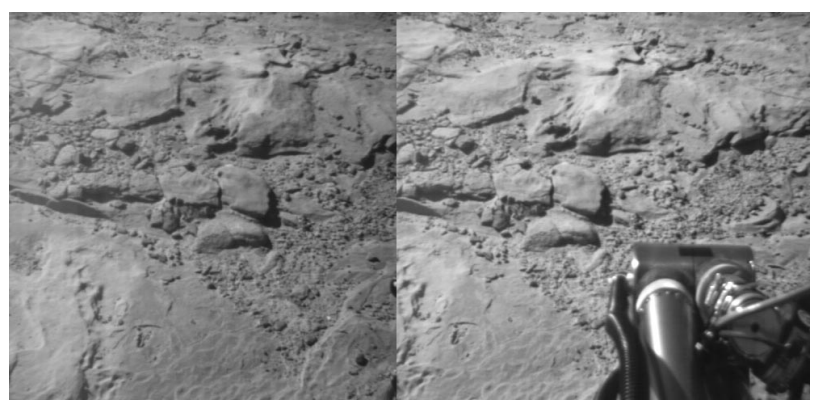

Figure 8: Stereo pair from the primary cameras

consists of a single grayscale image. Stereo pairs take a left image, then a right image, and send them joined together left-right in a single image (see Figure 8)

Panoramas are formed by moving the pan-tilt to a start location, digitizing the central column from the image, step- 


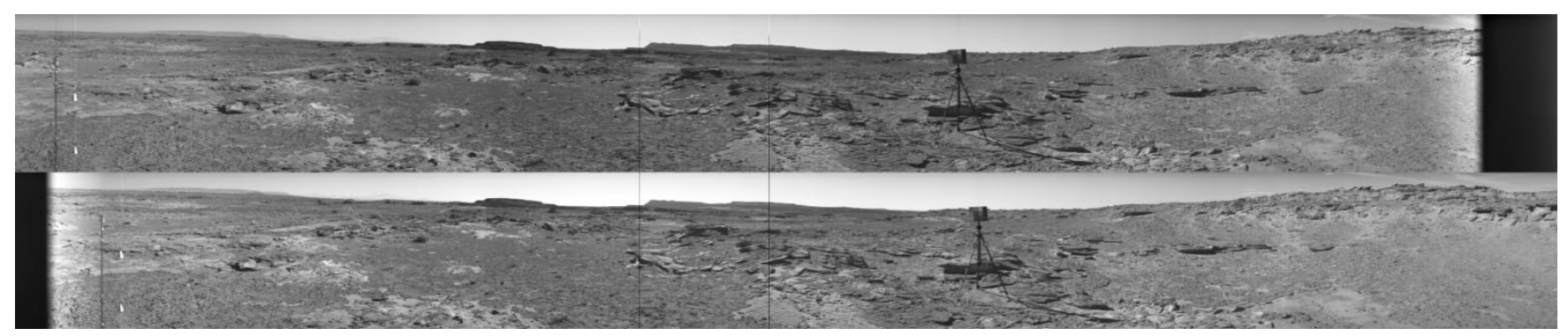

Figure 9: $263^{\circ}$ stereo panorama from landing site with windsock and color calibration targets.

ping one pixel width to the next pan position, and repeating until reaching the end location. This technique can generate a $318^{\circ}$ panorama with no lens distortion effects along the pan axis. Camera AGC changes are smoother and less distracting than when mosaicing individual images together. Stereo panoramas are done in the same fashion (see Figure 9).

Each image is sent back to the control station as a header with state information and a series of image packets. Because a packet may be lost, a simple acknowledgment from the control station indicates that a packet has been received. If the acknowledgment is not received within $3 \mathrm{sec}-$ onds, the packet is resent up to 5 times. Because of satellite delays $(600 \mathrm{~ms})$ and communication overhead, this resulted in a full size mono image taking 4 minutes to transmit from Arizona to ARC.

\subsection{Onboard computers}

In an enclosure on the rear pallet, a VME chassis houses the computers and interfaces for all onboard systems. A Motorola 68060 processor provides all computation. One 6 axis servo control board provides velocity control servo loops for the vehicle chassis. A 5 axis servo control board provides position control for the arm. A 4 port serial interface card talks to the compass/inclinometer, the arm force sensor, the pan-tilt controller, and a multi-drop serial control bus.

The multi-drop serial bus controls a series of simple digital and analog interface modules. These control power to the base, control power to the arm, control the carousel, control camera selection, control the filter wheel, and monitor the vehicle chassis angles.

An Arlan radio ethernet bridge communicates offboard at $500 \mathrm{kbps}$. The robot uses a $6 \mathrm{db}$ omni-direction antenna for uniform coverage. The stationary end of the link uses a $12 \mathrm{db}$ directional antenna to improve operating range and reduce multi-path effects.

\subsection{Vision-based navigation}

To reduce operator control cycles and improve navigational accuracy, a vision-based tracking system autonomously drives the robot to an operator designated natural feature (e.g. a rock outcropping) [Wettergreen 1997].

The feature tracking system uses a robust image correlator based on binary correlation of the sign of the difference of Gaussian of an image. As the robot moves, this correlator tracks a feature from frame to frame and performs stereo correlation to estimate the feature range. Input imagery comes from the stereo pair of cameras on the rover mast.
After a human operator designates a target feature, two control loops are activated to drive the robot to the feature. A gaze fixation loop correlates between previous and current images from one of the cameras, controlling the pan-tilt head to keep the target feature centered in the camera's field-ofview. A robot motion control loop correlates between left and right images, and uses the stereo range data in conjunction with the bearing data from the fixation loop to keep the vehicle driving to the feature. This control loop also halts the vehicle when the feature is within the desired distance.

\subsection{Remote control interface}

The Marsokhod remote control interface is made up of four subsystems: the Virtual Environment Vehicle Interface (VEVI), the rover manager, the rover operator, and the telemetry router (see Figure 10).

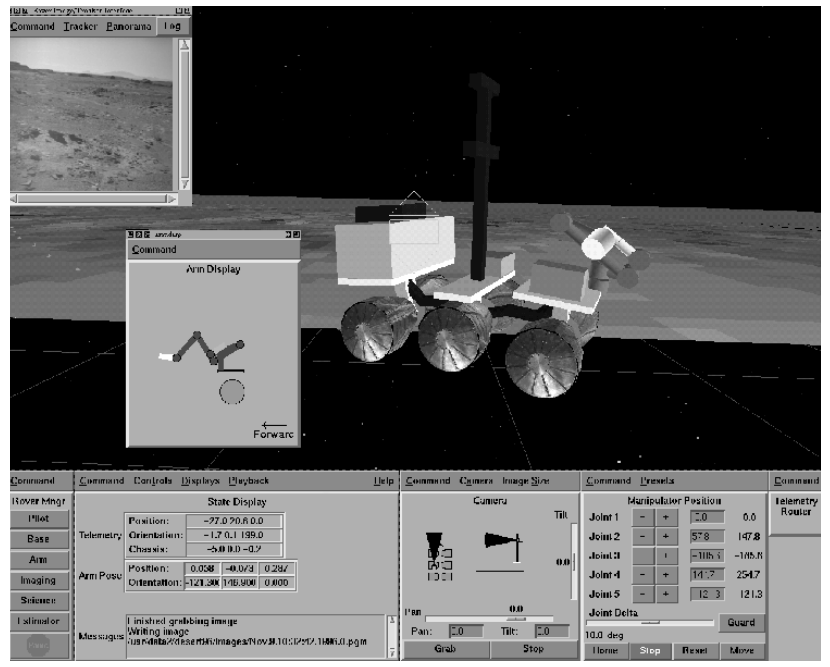

Figure 10: Remote control interface

VEVI is a real time, networked visualization system used to display rover state and local terrain. As the rover moves, VEVI shows the rover position and orientation, the articulation of the chassis, and the state of the arm in a 3D virtual world [Hine 1995].

The rover manager, rover operator, and telemetry router are written in the TCL/Tk interpreted scripting language. Using the built in widgets for buttons, entries, canvases, etc., a 2D interface controls all systems of the rover.

A single rover manager is the one connection from any number of "ground stations" to the rover. Several operator 
interfaces may be run at once, but all messages are routed through the manager. The rover manager can disable families of rover commands by simply not relaying those commands to the rover. The rover manager can also introduce a communications delay by holding on to a message for a specified amount of time before sending it out. In this way, we can simulate communications delays between Earth and Mars.

The rover operator consists of several windows that display rover telemetry and provide the controls for the various rover subsystems. The two main panels are the telemetry panel that displays rover state, trip distance, and warning messages, and the camera control panel that enables the user to control the pan/tilt head and grab images from the various cameras on-board. Several specialized interchangeable panels give the user access to two driving modes (gross motion and individual wheel control), three arm control modes (joint angles, jog, and cartesian), and control of the carousel. An image display/tracker control panel is available to display camera outputs and to provide the controls for the on-board visual tracking system. Additionally, two pop-up display panels are available to aid the operator in visualizing arm controls and chassis articulation.

A telemetry router is the single connection from the rover to any number of operator interfaces. All telemetry from the Marsokhod is sent through the telemetry router to its final destination.

All three interface subsystems automatically create logs. The rover manager logs all commands to the rover, the telemetry router logs all telemetry from the rover, and the rover operator logs comments and annotations made by the user and all imagery data (file names, time stamp, rover state at time of image grab).

As part of an outreach program, the IMG developed a textonly interface that could be used by school children to drive the rover and control its cameras. The participating Native American schools logged on to an IMG workstation and ran the text-only interface which gave them simple menus for controlling the rover direction, speed, and pan/tilt angles. They could see the results of their commands by watching as imagery and telemetry were updated on the IMG web site.

\section{Field test environment}

Our most recent field experiment using the Marsokhod took place near Tuba City on the Navajo reservation in the Painted Desert region of northern Arizona. The site was chosen for its sparse vegetation, varied geology, and Mars-like appearance. The test was run in November to avoid high temperatures.

The science team was told little about the location of the site (only that it was in Arizona) and nothing about the local geology. The few people who had been to the site (in order to select it) were placed in administrative roles and conveyed no knowledge of the site to the science team. Simulated descent images were taken from a helicopter several weeks prior to the field test. A simulated satellite image was provided from high altitude overflights done years earlier.

Because of limited availability of the science team, operations that would have taken weeks or months on Mars were compressed into 6 days of operations. A full resolution, color panorama was taken prior to the science test to save the 7 hours necessary to acquire and transmit it.

\subsection{Field operations}

The field site was 10 miles from the nearest town and 60 miles from a major city. No power or telephone service were available near the site. A rented recreational vehicle served as a operations truck and contained all operational and logistical equipment for the field test. Portable generators provided power for the operation truck and for the robot. A rented moving van provided overnight shelter for the robot from the weather and served as a workshop. Because of the age of the batteries in Marsokhod, it was run off of a tether from an easily moved generator for all field operations. The batteries provide heavy load power and the tether provides all standard operating power.

A commercial satellite link provided communications at $112 \mathrm{kbps}$ from the field site back to ARC. Cellular phones with high gain antennas aimed at the nearest cell in Utah provided voice communications for trouble shooting and ground truth support. The phone link was kept open during all operations. A person on the operations truck could talk to ARC via phone, talk to the field support team via hand held radios, and could monitor all data from the robot.

Field team operations ran from sunrise to a hour after sunset each day. Operation from ARC ran roughly 9-5 each day (AZ time). A geologist from Arizona State University took notes on what the robot was observing, took reference photographs, and prepared a detailed ground truth report after the test was complete.

\subsection{Online collaboration}

The ARC Marsokhod team developed a web site ${ }^{3}$ through which all mission data was available as soon as it was received at Ames. Ancillary information was also available for the science teams to assist in finding and processing the data and understanding the rover capabilities. Additionally, the web site provided public outreach on all aspects of the mission.

The original portable pixel map (PPM) format images from the robot are archived, and are also converted to GIF, JPEG, and TIFF formats for various image analysis tools. The header and telemetry information can be overlaid onto the image for printing and web site use.

Data available on the web site includes the following: guided tour of the web site; most recent images and telemetry (updated every minute); red-blue stereo images; air photos annotated with actual rover path; Quicktime VR panoramas of the science panorama images; mission logs; background information; control display screen shots; camera specifications; Marsokhod specifications; photos of people and equipment; animated image sequences from onboard cameras; press releases; test site general information; and the scientist briefing packet.

\section{3. http://img.arc.nasa.gov/marsokhod}




\subsection{Control center operations}

The science and rover operations at ARC was conducted in a small control room. The rover operators and mission scientists shared the space and interacted constantly. Silicon Graphics workstations provided all operational control and access to all the data. A menu driven interface was provided to access the different data analysis programs. Several image processing and display tools were provided: XV, PhotoShop, Netscape, and ImageMagick. Two Macintoshes were provided for access to NIH Image and PhotoShop.

Two workstations were designated for the rover operator and engineering support. Scientists were requested to stay out of this area to avoid distracting the rover operator. A second, large monitor of the rover operator's screen was placed in the science area to view live telemetry and images.

Three methods were used to view stereo images: LCD shutter glasses (Crystal Eyes) were used view a computer monitor in a special mode, red/blue anaglyphs were use with on screen display and color printouts, and a stereo air photo viewer was used to view printouts. The red/blue anaglyphs can be viewed on any monitor or on color print outs. The LCD glasses allow multiple people to view a single image on a specially equipped workstation. Manual processing steps were needed before any images could be viewed in stereo.

Several monochrome and color printers were made available in the control center or nearby for hard copy of images. The initial full color panorama was printed out at $10 \mathrm{ft}$ wide resolution and hung on one wall.

\subsection{Ancillary tests}

Some additional tests were performed to take advantage of the field site. These tests showed promise for future research but are only in preliminary development.

A deployable micro-rover was tested to investigate the potential for a small rover to assist as an imaging calibration target, an external inspection camera, a navigation reference, and a high risk explorer. Koala ${ }^{4}$ is a $32 \times 32 \times 20 \mathrm{~cm}, 6$ wheeled skid steered rover with simple onboard autonomy. For the field test it was equipped with a fixed monochrome camera which sent live video to the operations truck.

A haptic device recorded the frequency and amplitude of the needle sliding over the surface of the sample using a piezo vibration transducer. The forward motion of the needle is provided by the rotation of the carousel. A replay device allowed the remote scientist to feel by tactile sensation the texture of the sample recorded by the needle.

A simulation of the Pathfinder windsock experiment was performed to test imaging requirements. The rigid windsocks are displaced from a vertical orientation by air movement and imaged to measure this displacement and its direction. The objective was to get image data in realistic environments for later experiments with image compression levels.

4. Koala is made by the K-team (http://www.kteam.com) in collaboration with the Swiss Federal Institute of Technology of Lausanne (EPFL).

\section{Science experiments}

The science teams working at NASA Ames Research Center had three specific goals for the 6 days of field testing in Arizona. The primary goal was to establish the general geology and biology of the field test site. The second goal was to test methods and techniques to be used with the Mars Pathfinder mission and the Sojourner rover in July 1997. The third goal was to try out a mode of exploration based on rapid exploration.

To accomplish the science goals, the field test was split into two day phases: Pathfinder simulation, general exploration, and rapid exploration. Some scientists participated in the team for more than one phase. Since the Pathfinder lander was not equipped with a descent camera, the Pathfinder team went first and was not given descent images. The second and third phases were given simulated descent imagery at the start of operations. This allowed the team to select not only nearby science sites, but areas outside the "landing area" that looked significant. The third phase team came with a path sequence planned before arriving at Ames using only descent imagery. The path was executed regardless of what the team learned at each site.

The robot was carried back to the "landing site" at the start of each phase. Onboard odometry show that the rover travelled a total of $469 \mathrm{~m}$ during the science test (see Figure 11).

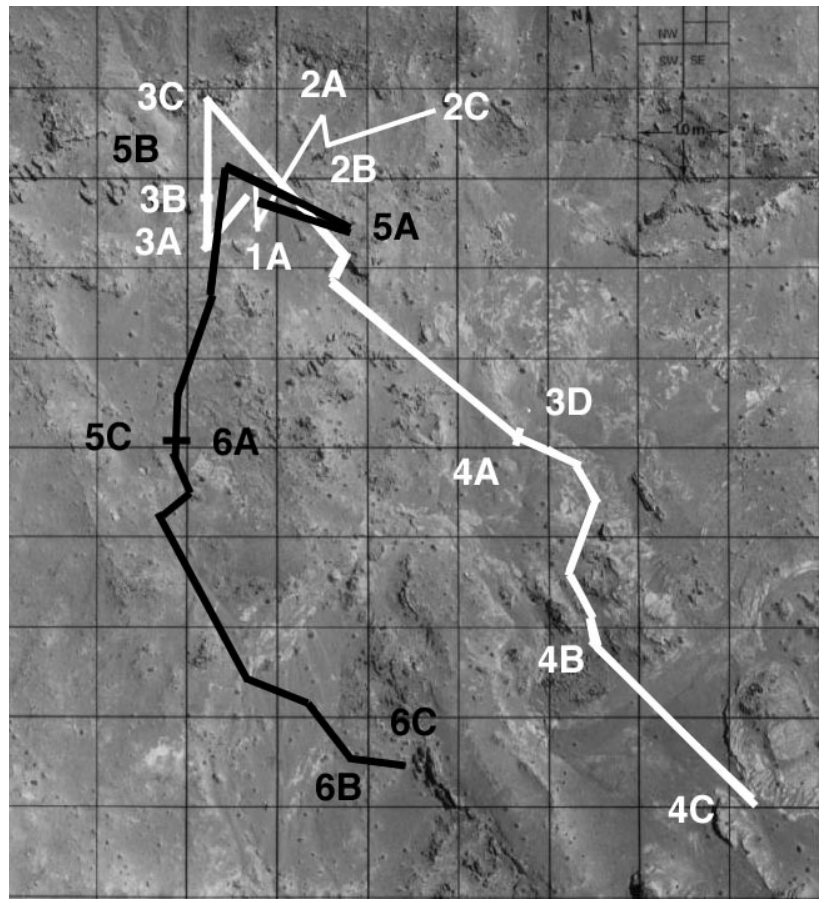

Figure 11: Rover paths on a 10m grid

The robot was driven back from the last science stop toward the landing site with a 6 minute time delay enabled. The longest single move was $45.3 \mathrm{~m}$.

\subsection{Science team organization}

Initially, the science team organized itself as a team lead, CapCom, scribe, and an analyst and processor for trafficability and for site analysis. However, the extensive terrain capa- 
bilities of Marsokhod made trafficability a simple issue that each person could understand. The distinction between analyst and processor also proved to be unnecessary. Members did their own processing and helped each other as needed.

The science members were supposed to talk to the rover operator/pilot through one designated person that the science team referred to as "CapCom." This formal structure was often skipped, and the science team members talked directly to the rover operator.

The science team used labeled descent photos with $2 \mathrm{~m}$ or $10 \mathrm{~m}$ grids to communicate positions (see Figure 11). They would mark a target destination on a gridded photo and give that to the rover operator. It let the rover pilot focus on driving without having to continually interact with the science team to make sure the rover was heading to the correct location. The field reference geologist used the same gridded photos for preparing the ground truth notes.

Having little formal structure to the team promoted free exchange of ideas, but was occasionally chaotic.

\subsection{Rover operations}

The rover operator is an engineer trained in all the details and limitations of operating Marsokhod. The operator takes science sampling objectives and controls the robot to get the desired information.

To navigate to a new destination, the operator would take a high resolution stereo image toward the destination to establish context of the new area, designate a clear target near the desired position, and use the vision-based tracking to drive there. The position and orientation of the rover was then verified using the cameras and descent photos. The tracking cycle may be repeated if the rover was very far from the destination or manual drive commands may be used for final moves to the desired destination.

Depending on the nature of the science objectives, various types of images would be taken.

For an arm placement, the rover was first moved into a position that can reach the target and has good visibility from the cameras. The arm was then moved to a position above the target. The main and front pallet cameras can be used to align the position and orientation of the arm above the target. The close-up imagers may be useful, but are usually too out of focus for approach alignment. Then a guarded move would place the arm in contact with the surface. If the orientation of the arm is wrong, the close-up imager will be out of focus. The arm then had to be raised, re-oriented, and replaced. Samples would be placed in the storage container using preset positions. The arm was returned to the "home position" before any further driving.

\subsection{Robotic field exploration methods}

Several robotic exploration methods emerged during the testing. Many limitations found early on were fixed during the course of the test. Other limitations in the capabilities of the robot define the areas of future research in remote surface exploration.

Teams with descent imaging used them to communicate locations and discuss theories. The high resolution panorama was used for up to date information and was the only source of context for the Pathfinder team.

Comparison of half and full resolution images shows that many conclusions cannot be made with the lower resolution images alone. Single band images from the multi-spectral imager were sharper and higher resolution than the main stereo cameras. However, the assembled full color images had color alignment problems that lowered the overall spatial resolution. Infrared band images lost most of their resolution due to poor focus.

Mono images were used as the primary source for scientific analysis. Color images and stereo viewing provide the understanding of features and geologic context. Then detailed analysis is done on individual images.

Different people had specific preferences for viewing stereo images. Some people were annoyed by the flicker from the LCD glasses. Some people were annoyed by the cross over effects in the red/blue anaglyphs.

The stereo cameras on the front pallet used for arm placement sometimes provided better close up views than the main cameras. However, the shadows from the rover and the image quality on these particular cameras greatly limited their use. Fast access to stereo viewing would have made them more useful for arm placements.

The use of paper prints of images varied from team to team. In particular, the Pathfinder team (which didn't have descent photos) needed many high resolution print outs of sections from the main panorama to aid in planning.

Locating the rover position and orientation from imaging and the descent photos was critical and often difficult. Imaging was taken around the robot and matched to descent images. At one point, a terrain discrepancy more than tripled the time to verify position.

Several techniques were used to help determine surface characteristics. The simplest is to look at the tracks behind the rover. The surface will show the effects caused by three wheels travelling over it. By driving forward then reversing, the effects of a single set of wheels can be observed. Turning in place shows the effects of having wheels dragged across the surface.

Additional surface experiments were done using the clamshell with the instrument carousel in contact with the ground. The simplest was to close-up image the surface, close the clam-shell, open the clam-shell, and then image again. Hard surfaces would not be marked. Medium hardness surfaces would have marks from the edges of the clam-shell. Loose surfaces would be disturbed by clam-shell closing through it

Another clam-shell technique was to close-up image the site, close the clam-shell, remove (and optionally store) the sample, replace the arm in the same position, and re-image the site. The hole left from sampling would show soil characteristics.

The close-up cameras provided much needed detail about surfaces, but were limited by the narrow depth of field. Only flat surfaces could be entirely within the depth of field. This was further hampered by difficulties getting the instrument carousel flat against a surface.

A number of data organization issues limited ease of access to the rover data. The web interface was little used because it was simply too slow at finding and bringing up 
images. Photoshop was limited by the large number of file names to scroll through to open an image and by the limited number of machines licensed to run it. XV was unfamiliar to most people, but became the most used interface due to its intuitive controls and fast response. Difficulties with file naming conventions and cross referencing of images in the logs could only be partially remedied during the test to avoid inconsistencies in the data archive.

The scientists were impatient both with accessing previous data as well as taking new images. The delays slowed down their ability to formulate theories about what they were seeing and to have discussions about them.

One scientist did work remotely, using the web interface for data and the conference phone to interact with the rest of the science team.

\section{Discussion}

The most recent Marsokhod field experiment revealed many areas for research to improve remote science exploration efficiency and effectiveness.

The resolution of the cameras, $1 \mathrm{mrad} / \mathrm{pixel}$, was not satisfactory for remote geology. Scientists felt that they could not see enough geologic structure from a distance and that they could not resolve surface features close up. In one anecdotal view, the Marsokhod "missed 90\%" of the interesting geology it encountered. An expert image processor can enhanced versions of images to make particular features clearer.

Color imaging is vital for establishing geologic context. Multi-spectral imaging, if used, must be carefully configured to discriminate specific mineral comparisons.

Panoramic images provide essential context for other data. Using fully encompassing panoramas, scientists were able to localize (determine the position and orientation of the robot), to more fully characterize the gross geology of the site, and to plan areas for Marsokhod to investigate and/or traverse.

Range measurements in stereo image pairs need to be readily available and accurately calibrated. Alternatively, a 3D surface model constructed from the stereo images should incorporate tools for making measurements.

Manual or automated techniques for tracking horizon features could greatly speed up the ability to verify position and orientation.

The ability of the carousel to image and sample at the same site proved very useful and enabled several useful investigation techniques. The ability of the arm to land precisely on a designated target will require further development, both in methods of defining the target and in the ability to automatically approach, contact and settle on the target.

Access to the field test data should be re-structured to promote fast, understandable access. Pre-test training sessions would reduce confusion and identify issues regarding units, coordinate frames, and other conventions. The team structure of the science and engineering teams continues to improves as we gain more experience. Fast access to high resolution, well understood data enables strong collaboration.

The rapid development techniques used in the user interface enabled many adjustments to the needs of the science team without creating limitations or reliability concerns.
Feedback from the ground truth scientists on a daily or test phase basis would have helped the scientists understand how much can be inferred from the robot imagery and what types of misconceptions tend to arise.

\section{Summary}

Field experiments with the Ames Marsokhod have helped to understand the requirements for doing science in a place where people have never been. It is clear that mobility is a very powerful part of understanding the geology of a site. A number of areas for future research are revealed by system limitations. Many of the successful techniques suggest areas to develop onboard autonomy.

\section{Acknowledgments}

This work was supported by the NASA Space Telerobotics Program. Dr. Carol Stoker provided additional funding and leadership for the field test.

This represents the efforts of the entire IMG team: Maria Bualat, Dan Christian, Mike Costa, Dan Daily, Lorenzo Flueckiger, Aaron Kline, Linda Kobayashi, David Hasler, Phil Hontalas, Michael Li, Cesar Mina, Pat Payte, Daryl Rasmussen, Kurt Schwehr, Michael Sims, Mike Solomon, Geb Thomas, Hans Thomas, Deanne Tucker, Dave Wettergreen, and Eric Zbinden. In additional many people at ARC contributed to setting up and performing the science experiment: Nathalie Cabrol, Jack Farmer, Edmund Grin, Virginia Gulick, Rags Landheim, Ted Roush, and Jeff Moore.

Additional thanks go to many people at the USGS and Ron Greeley, Michael Kraft, Dave Nelson, and Jim Rice from Arizona State University for helping with logistic and scientific support. Many thanks to George McGill, Henry Moore, and Robert Reid for documenting the results of the science teams and reviewing the field test. Special thanks to the Navajo nation for their hospitality, support, and enthusiasm (and Navajo Tacos).

\section{References}

[Kermurdjian 1992] A. Kermurdjian, V. Gromov, V. Mishkinyuk, V. Kucherenko, and P. Sologub. Small Marsokhod Configuration. Proceedings of the 1992 IEEE International Conference on Robotics and Automation, May 1992.

[Hine 1995] Butler Hine and Phil Hontalas. VEVI: A Virtual Environment Teleoperations Interface for Planetary Exploration. In SAE 25th International Conference on Environmental Systems, San Diego, CA, July 1995.

[Wettergreen 1997] David Wettergreen, Hans Thomas, and Maria Bualat. Initial results from vision-based control of the Ames Marsokhod rover. In IEEE International Conference on Intelligent Robots and Systems, Grenoble, France, September 1997. 